\title{
Semantic cut elimination in the Intuitionistic Sequent Calculus
}

\author{
Olivier Hermant \\ Projet LogiCal \\ Pôle Commun de Recherche en Informatique du plateau de Saclay, CNRS, École \\ Polytechnique, INRIA, Université Paris-Sud. \\ ohermant@pauillac.inria.fr, http://pauillac.inria.fr/ ohermant
}

\begin{abstract}
Cut elimination is a central result of the proof theory. This paper proposes a new approach for proving the theorem for Gentzen's intuitionistic sequent calculus LJ, that relies on completeness of the cutfree calculus with respect to Kripke Models. The proof defines a general framework to extend the cut elimination result to other intuitionistic deduction systems, in particular to deduction modulo provided the rewrite system verifies some properties. We also give an example of rewrite system for which cut elimination holds but that doesn't enjoys proof normalization.

Keywords: intuitionistic sequent calculus, Kripke Structure, semantic, deduction modulo, cut admissibility, cut elimination property ${ }^{1}$
\end{abstract}

\section{Introduction}

Since Gentzen's result [1], the cut elimination theorem has been a central result of Proof Theory. Proving the cut elimination theorem is the key to the good properties of deduction systems, such as consistency, or the disjunction and the witness property for the intuitionistic framework. It allows also to prove the decidability of some logical fragments (as the propositional case), and is essential for proving completeness of proof search methods such as tableaux or resolution $[2-4]$.

Two main approaches can be used to establish the result. One way is a syntactic one, proving termination of a certain cut-elimination process, as in the original proof of Gentzen [1]. A modern way to prove the result uses proof terms [5] and reducibility method.

The other way is to prove the admissibility (or redundancy) of the cut rule [6-8], proving completeness of the cut-free calculus with respect to some notion of model. This is known since Beth, Hintikka and others [9], and this has been recently used by De Marco and Lipton [10] to prove cut elimination of the Intuitionistic Higher-Order Logic, and by Okada [11] for intuitionistic Linear Logic (first and higher-order).

\footnotetext{
1 (C) Springer-Verlag
} 
An interesting field of research is to try to understand the links between these two methods. In particular, one may ask if all formalisms verifying cut admissibility are normalizing under proof reduction.

A first difficulty for this study is that intuitionistic logic seems to be a better framework for proof normalization, whereas classical logic is easier to use when dealing with semantic methods. There are two manners to bridge the gap: either study proof normalization for a classical logic, either, as we do here, establish semantic methods for the intuitionistic logic.

In this paper, we thus describe a semantic method to prove cut admissibility in the intuitionistic sequent calculus. Although the result is not new, the method seems not to have been used yet. Moreover, it extends easily to sequent calculus modulo several congruences, as we will see in the last part of the paper. This is an important extension for two reasons. First, it shows cut-elimination for many axiomatic theories, without considering ad-hoc axiomatic cuts. Then, Deduction Modulo is a good framework to understand the links between semantic and syntactic approaches, since Dowek and Werner have defined in [5] general syntactic methods for proving termination of proof reduction, based on pre-models and reducibility candidates.

Our model construction is obtained by transforming Gödel's completeness theorem for first-order classical logic. We prove completeness of the cut-free sequent calculus with respect to some notion of model. The construction is similar in many aspects to Gödel's, but differs on several important points. First, we consider here intuitionistic logic instead of classical logic, thus our models will have a different form. Then - and this is the most important point - we consider a cut-free calculus. This leads to many technical difficulties. In particular it requires to introduce new definitions of consistency and completeness of a theory.

Unlike classical logic, intuitionistic logic has many different notions of models, among which Kripke Structure [12] and Heyting Algebras [13]. Recently, an extension of Heyting Algebra have been used by De Marco and Lipton [10] to prove cut redundancy for Intuitionistic Higher-Order Sequent Calculus. Okada, in [11], uses phase semantics, that reduce to Heyting Algebra in the Intuitionistic Logic subcase. In this paper, we will use Kripke Structure. We believe that Kripke Structures lead to much simpler proofs, in particular they seem to extend rather straightforwardly techniques already used in classical logic ([14, 8]).

In the last section, we discuss shortly the extension of the result to the Deduction Modulo. An example is given, where the cut-elimination proof appears to be a very simple modification of the former. We also present a terminating, confluent rewrite system such that deduction modulo this rule enjoys cut-elimination, although the cut-elimination process using reduction of proof-terms fails [5].

In the later, we will consider, unless specified, the cut-free intuitionistic sequent calculus, this is the calculus as defined in figure 1 minus the cut rule. In a sequent $\Gamma \vdash P, \Gamma$ is a finite multiset of proposition (a proposition can appear several times). So we can always find a fresh constant (provided the language has a countable set of constants) to introduce in the rules $\forall$-right and $\exists$-left. 


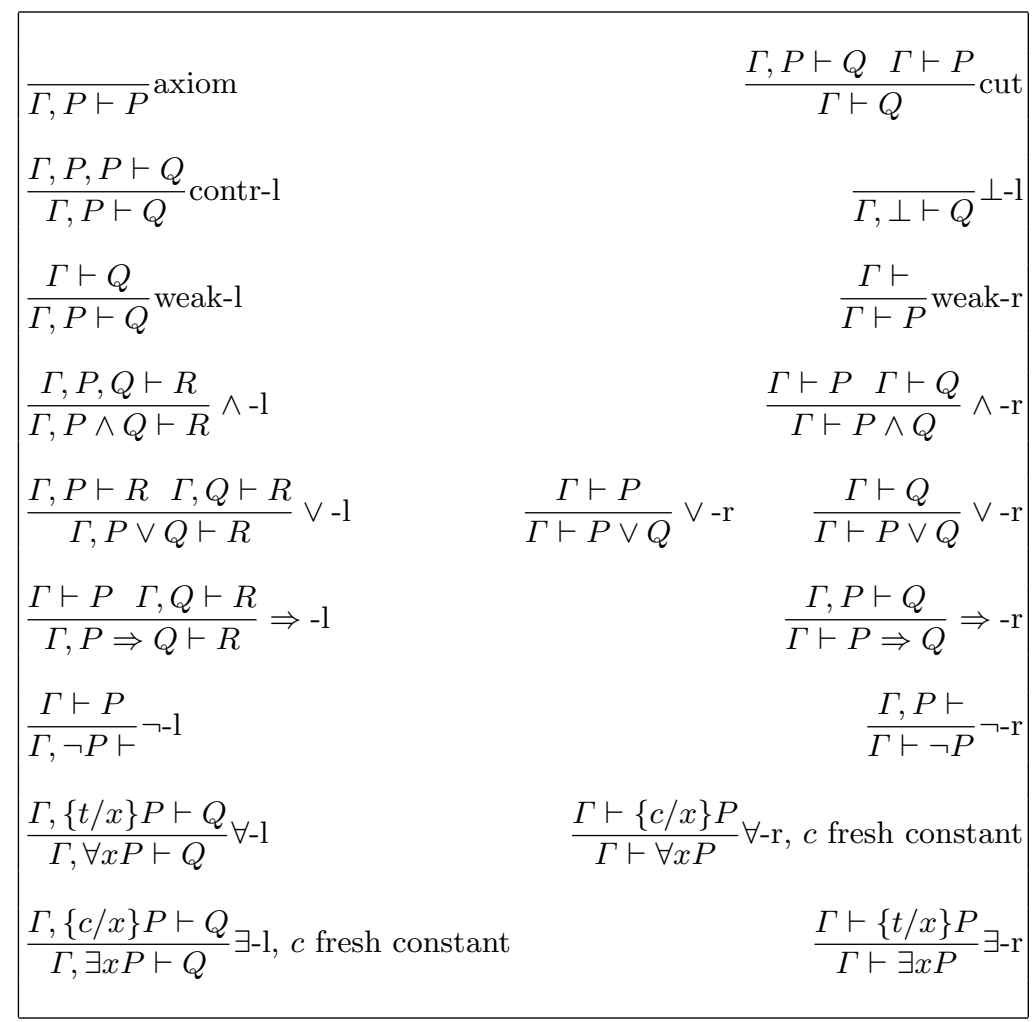

Fig. 1. Deduction rule of intuitionistic sequent calculus (with the cut rule)

These two rules are usually (equivalently) formulated with fresh variables instead of constants. Here we prefer this formulation, that avoid considerations over $\alpha$-equivalence of propositions.

To recall that we are working in the cut-free sequent calculus, we will write a sequent $\Gamma \vdash^{c f} P$

\section{Definitions}

In [10], De Marco and Lipton discuss the reason why Henkin's completion process [12] fails when we disallow the use of the cut rule. This is the case because the completion process is done with a heavy use of the cut rule. Then, the authors discard the usual completeness notion, and build downward complete sets (with respect to the subformula property), in defining a very nice tableau construction for intuitionistic logic.

Here, we propose a different approach, that keeps the notion of a complete theory. In fact, we adapt the notion of complete theory to the cut-free calculus in a very simple way. The reader can check that when the cut rule is allowed, 
the two completeness notions are equivalent. But when it is not, the two notions split.

Our construction, and the completion process that follows have the advantage to preserve the maximality of the constructed theories. Moreover, they stick to more usual completeness construction [12] used to define the semantic of sequent calculi (with the cut rule).

We also need a larger understanding of completeness and consistency, because we are in an intuitionistic framework. So, we are led to define $A$-consistency and $A$-completeness, where $A$ is a formula. From these definitions, it becomes simple to prove the completeness theorem, following the lines of Gödel's proof, applying it to Kripke Structures.

Definition 1 (A-consistency). Let $A$ be a proposition. A set of propositions (theory) $\Gamma$ is said to be A-consistent iff $\Gamma \nvdash^{c f} A$.

Definition 2 (A-completeness). Let $A$ be a proposition. A set of propositions (theory) $\Gamma$ is said to be A-complete iff for any proposition $P$, either $\Gamma, P \vdash^{c f} A$, or $P \in \Gamma$.

Definition 3 (A-Henkin witnesses). Let $A$ be a proposition. A set of propositions (theory) $\Gamma$ is said to admit A-Henkin witnesses if for any proposition of the form $\exists x P$ such that $\Gamma, \exists x P \nvdash \nvdash^{c f} A$, there exists a constant $c$ such that $\{c / x\} P \in \Gamma$.

These definitions are different from from those used in the classical case [14], because we are now in the intuitionistic framework: in particular, we don't have symmetry between the left and the right parts of a sequent, so we lose symmetry between $\forall$ and $\exists$ quantifiers, and we can't have Henkin witnesses for both of them. Another point is that instead of considering only consistency, we have to consider $A$-consistency, so sets of propositions become smaller, although they still possess all the good properties we need, as shown in section 3.2

\section{Definition 4 (Kripke Structure).}

A Kripke Structure $\mathcal{K}$ is a quadruple $\langle K, \leq, D, \Vdash\rangle$, such that $K$ is a set (the set of nodes, or worlds), $\leq$ is a partial order on $K, D$ a function (called the domain) from $K$ to non empty sets, that is monotone w.r.t. $\leq$ (if $\alpha \leq \beta$ then $D(\alpha) \subseteq D(\beta))$. And $\Vdash$ is a relation between elements $\alpha \in \bar{K}$ and the closed propositions over $D(\alpha)$, such that:

1. for any $A\left(x_{1}, \ldots, x_{n}\right)$ atomic, any worlds $\alpha \leq \beta$, any $a_{1}, \ldots, a_{n} \in D(\alpha)$, $\alpha \Vdash A\left(a_{1}, \ldots, a_{n}\right)$ implies $\beta \Vdash A\left(a_{1}, \ldots, a_{n}\right)$

2. $\alpha \Vdash A \vee B$ iff $\alpha \Vdash A$ or $\alpha \Vdash B$.

3. $\alpha \Vdash A \wedge B$ iff $\alpha \Vdash A$ and $\alpha \Vdash B$.

4. $\alpha \Vdash A \Rightarrow B$ iff for any $\beta \geq \alpha, \beta \Vdash A$ implies $\beta \Vdash B$.

5. $\alpha \Vdash \neg A$ iff for any $\beta \geq \alpha, \beta \nVdash A$.

6. $\alpha \Vdash \exists x A$ iff there exists $a \in D(\alpha)$ such that $\alpha \Vdash\{a / x\} A$.

7. $\alpha \Vdash \forall x A$ iff for any $\beta \geq \alpha$, for any $a \in D(\beta), \beta \Vdash\{a / x\} A$. 
With respect to Kripke Structures, we should first prove soundness of the Intuitionistic Sequent Calculus with cut.

Theorem 5 (Soundness). Let $\Gamma$ be a set of propositions, and $P$ be a proposition. If $\Gamma \vdash P$ (with possible use of the cut rule), then for any Kripke Structure $\langle K, \leq, D, \Vdash\rangle$, for any node $\alpha \in K$, if $\alpha \Vdash \Gamma$ then $\alpha \Vdash P$.

We write $\Gamma \models P$ if $P$ is valid at any node that validates $\Gamma$.

Proof. We check that all the derivation rules are valid as in [15]. The result holds also for the cut-free sequent calculus, but this is not relevant here.

The difficult part is to prove the converse, namely the completeness theorem. In our case, we have to prove completeness of the cut-free calculus with respect to Kripke Structures.

\section{Completion of a theory and basic results}

First, given a theory $\mathcal{T}$ and a proposition $A$ such that $\mathcal{T}$ is $A$-consistent, we describe how to get an $A$-complete, $A$-consistent set $\Gamma$ containing $\mathcal{T}$, admitting $A$-Henkin witnesses. Then, we will describe the properties of $\Gamma$.

\subsection{Completion}

Let $\mathcal{L}$ be a language, $\mathcal{T}$ a theory in $\mathcal{L}$, and $A$ a proposition such that $\mathcal{T} \nvdash^{c f} A$. We consider an infinite set of constants $\mathcal{C}$ disjoint from $\mathcal{L}$, and we define $\mathcal{L}^{\prime}=\mathcal{L} \cup \mathcal{C}$.

We consider an enumeration of the propositions of $\mathcal{L}^{\prime}: P_{0}, \ldots, P_{n}, \ldots$ and we let $\Gamma_{0}=\mathcal{T}$. We define $\Gamma_{n}$ by induction:

- if $\Gamma_{n}, P_{n} \nvdash^{c f} A$ and $P_{n}$ is not of the form $\exists x Q$ we let $\Gamma_{n+1}=\Gamma_{n} \cup\left\{P_{n}\right\}$.

- if $\Gamma_{n}, P_{n} \nvdash^{c f} A$ and $P_{n}$ is of the form $\exists x Q$, we let $\Gamma_{n+1}=\Gamma_{n} \cup\left\{P_{n},\{c / x\} Q\right\}$, where $c \in \mathcal{C}$ is a constant that doesn't occur in $\Gamma_{n}$.

- otherwise we let $\Gamma_{n+1}=\Gamma_{n}$

Notice that in the first case, if $P_{n}$ is of the form $\exists x Q$, we have $\Gamma_{n},\{c / x\} Q \nvdash^{c f}$ $A$ since $c$ is fresh. So we don't lose the $A$-consistency of $\Gamma_{n+1}$.

Finally, we let $\Gamma=\bigcup_{i=0}^{\infty} \Gamma_{i}$.

\subsection{Properties of the completed theory}

Proposition 6. $\Gamma$ is A-consistent, A-complete, and admits A-Henkin witnesses.

Proof. Let's see the proof for $A$-completeness: suppose $\Gamma$ is not $A$-complete, so there exists a proposition $P$ such that $\Gamma, P \nvdash^{c f} A$ and $P \notin \Gamma$. By the former enumeration, there exists a $n$ such that $P=P_{n}$. We have $\Gamma_{n} \subseteq \Gamma$, so $\Gamma_{n}, P_{n} \nvdash^{c f}$ $A$. We get a contradiction, since by construction $P_{n} \in \Gamma_{n+1} \subseteq \Gamma$.

The two other properties are proved in the same way. 
An important property of any $A$-consistent, $A$-complete theory admitting $A$-Henkin witnesses is that it enjoys some form of the subformula property.

Proposition 7. Let $A$ be a proposition and $\Gamma$ an $A$-complete, $A$-consistent set of propositions that admits A-Henkin witnesses. Then:

1. if $P \wedge Q \in \Gamma$ then $P \in \Gamma$ and $Q \in \Gamma$

2. if $P \vee Q \in \Gamma$ then $P \in \Gamma$ or $Q \in \Gamma$

3. if $\exists x P \in \Gamma$ then $\{c / x\} P \in \Gamma$ for some $c$

4. if $\forall x P \in \Gamma$ then $\{t / x\} P \in \Gamma$ for any $t$

5. if $P \Rightarrow Q \in \Gamma$ then either $Q \in \Gamma$, either $\Gamma \nvdash^{c f} P$

6. if $\neg P \in \Gamma$ then $\Gamma \nvdash^{c f} P$

7. if $\Gamma \nvdash^{c f} P \wedge Q$ then $\Gamma \nvdash^{c f} P$ or $\Gamma \nvdash^{c f} Q$

8. if $\Gamma \nvdash^{c f} P \vee Q$ then $\Gamma \nvdash^{c f} P$ and $\Gamma \nvdash^{c f} Q$

9. if $\Gamma \nvdash^{c f} \exists x P$ then for any term $t, \Gamma \nvdash^{c f}\{t / x\} P$

10. if $\Gamma \nvdash^{c f} P \Rightarrow Q$ then $\Gamma, P \nvdash^{c f} Q$

11. if $\Gamma \nvdash^{c f} \neg P$ then $\Gamma, P \nvdash^{c f}$

Proof. It relies essentially on the arguments that $\Gamma$ is $A$-complete, $A$-consistent, admits $A$-Henkin witnesses, and on the fact that we can use in a reversed way the rules of sequent calculus of figure 1 .

Let's see some examples:

- 3 is the $A$-Henkin witnesses property.

- In $5, P \Rightarrow Q \in \Gamma$ means in particular that $\Gamma, P \Rightarrow Q \nvdash^{c f} A$. We can not have at the same time $\Gamma, Q \vdash^{c f} A$ and $\Gamma \vdash^{c f} P$ (otherwise we could apply $\Rightarrow$-left rule). So, we have either $Q \in \Gamma$ (by $A$-completeness), or $\Gamma \nvdash^{c f} P$.

Notice that there are already links with Kripke Structures in this definition: at point $5, \Gamma \nvdash^{c f} P$ can be understood as the following: we can (in a richer language) complete $\Gamma$ in $\Delta, P$-complete, $P$-consistent, and that admits $P$-Henkin witnesses, in the same way as in section 3.1. So proposition 7 gives us a very easy way to construct a Kripke Structure, ordered by inclusion. This will be the object of next section.

\section{Completeness theorem and Cut Redundancy}

We are now ready to prove the completeness theorem. In fact, we will prove another equivalent formulation.

Theorem 8 (Completeness). Let $\mathcal{T}$ be a theory and $A$ a proposition, both expressed in some language $\mathcal{L}_{0}$.

If $\mathcal{T} \nvdash^{c f} A$ then there exists a Kripke Structure, and a world $\alpha$ such that $\alpha \Vdash \mathcal{T}$ and $\alpha \nVdash A$

Proof. First consider $\mathcal{C}_{n}$ a countable family of countable sets of new constants. We form the family of languages $\mathcal{L}_{n+1}=\mathcal{L}_{n} \cup \mathcal{C}_{n}$.

In the rest of the proof, we consider the Kripke Structure defined as follows: 
- $K=\left\{\Gamma \mid B\right.$-complete, $B$ consistent, $B$-Henkin, expressed in $\mathcal{L}_{i}$ for some $i$ and $\left.B \in \mathcal{L}_{i}\right\}$

- the order over $K, \leq$ is the large inclusion $\subseteq$

- $\mathcal{D}(\Gamma)$ is the set of closed terms of the language $\mathcal{L}_{i}$ in which is expressed $\Gamma$.

- the forcing relation $\Vdash$ defined by induction on the size of propositions. For atomic propositions we let $\Gamma \Vdash C$ iff $C \in \Gamma$. We extend this forcing relation to non atomic propositions thanks to the clauses $2-7$ of definition 4 .

It still remains to be checked that the first clause of the forcing relation holds: for any atom $C$, if $\Gamma \Vdash C$, let $\Delta \supseteq \Gamma$, we have to show that $\Delta \Vdash C$. This is immediate since $C \in \Delta$, so we straightforwardly use the forcing relation definition. Finally, we have checked all the clauses, and $\Vdash$ is a forcing relation.

By the completion procedure, we know the existence of a world $\Gamma$, expressed in $\mathcal{L}_{i}$, such that $\mathcal{T} \subset \Gamma$, and $\Gamma$ is $A$-consistent, $A$-complete and admits $A$-Henkin witnesses. It remains to prove that $\Gamma \Vdash \mathcal{T}$ and $\Gamma \nVdash A$. More generally, we will prove the following:

For any proposition $P$, for any world $\Gamma, P \in \Gamma$ implies $\Gamma \Vdash P$ and $\Gamma \nvdash^{c f} P$ implies $\Gamma \nVdash P$.

By an induction on the size of the proposition $P$ :

- the atomic case is immediate: if $A \in \Gamma$ so $\Gamma \Vdash A$. And if $\Gamma \nvdash^{c f} A$, then $A \notin \Gamma$, so by definition of the forcing relation $\Gamma \nVdash A$.

- if $A \vee B \in \Gamma$, we use proposition 7 and get $A \in \Gamma$ or $B \in \Gamma$, hence by induction hypothesis $\Gamma \Vdash A$ or $\Gamma \Vdash B$. Thus $\Gamma \Vdash A \vee B$.

if $\Gamma \nvdash^{c f} A \vee B$, by proposition 7, we have $\Gamma \nvdash^{c f} A$ and $\Gamma \nvdash^{c f} B$, so by induction hypothesis $\Gamma \nVdash A$ and $\Gamma \nVdash B$, hence $\Gamma \nVdash A \vee B$.

- if $A \Rightarrow B \in \Gamma$, let $\Delta \supseteq \Gamma$. Obviously $A \Rightarrow B \in \Delta$. By proposition 7, either $\Delta \nvdash^{c f} A$, either $B \in \Delta$. If the former holds, by induction hypothesis, we must have $\Gamma \nVdash A$. In the other case, we have $\Delta \Vdash B$ by induction hypothesis. So in both cases: $\Delta \Vdash A$ implies $\Delta \Vdash B$.

if $\Gamma \nvdash^{c f} A \Rightarrow B$, then by proposition 7 , we have $\Gamma, A \nvdash^{c f} B$. Let $\mathcal{L}_{j}$ the language in which is expressed $\Gamma$. By the completion procedure of section 3.1 , we can define in the language $\mathcal{L}_{j+1}$ a theory $\Delta \supseteq \Gamma$ that is $B$-consistent, $B$-complete and admits $B$-Henkin witnesses. This $\Delta$ is also a world of the Kripke Structure considered. And by induction hypothesis, $\Delta \Vdash A$ and $\Delta \nVdash$ $B$, so we must have $\Gamma \nVdash A \Rightarrow B$.

- if $\exists x P \in \Gamma$, then by the Henkin witnesses property, we have $\{c / x\} P \in \Gamma$, so by induction hypothesis, $\Gamma \Vdash\{c / x\} P$, and then $\Gamma \Vdash \exists x P$.

if $\Gamma \nvdash^{c f} \exists x P$, then, for any term $t, \Gamma \nvdash^{c f}\{t / x\} P$, by proposition 7 . So for any $t, \Gamma \nVdash\{t / x\} P$ by induction hypothesis. Hence $\Gamma \nVdash \exists x P$.

- if $\forall x P \in \Gamma$, then for any $\Delta \supseteq \Gamma, \forall x P \in \Delta$, and we use the same arguments as in the previous case to prove that $\Delta \Vdash\{t / x\} P$ for any $t$. So $\Gamma \Vdash \forall x P$. If $\Gamma \nvdash^{c f} \forall x P$, then let $\mathcal{L}_{i}$ the language of $\Gamma \cup\{\forall x P\}$. Let $c \in \mathcal{C}_{i}$. $c$ is fresh w.r.t $\Gamma$ and $P$ by construction of the set $\mathcal{C}_{i}$. So, we have $\Gamma \nvdash^{c f}\{c / x\} P$. By 
the completion procedure of 3.1, we get the existence of a world $\Delta,\{c / x\} P$ complete, $\{c / x\} P$-consistent admitting $P$-Henkin witnesses. So by induction hypothesis, $\Delta \nVdash\{c / x\} P$, hence $\Gamma \nVdash \forall x P$.

- the other cases are treated in a similar way.

As a corollary, we get the cut-elimination theorem:

Theorem 9 (Cut-elimination). If $\Gamma \vdash P$, then $\Gamma \vdash^{c f} P$.

Proof. Proof: Suppose $\Gamma \vdash P$. By soundness, $\Gamma \models P$, so there is no node $\alpha$ of any Kripke Structure such that $\alpha \Vdash \Gamma$ and $\alpha \nVdash P$. Hence by the completeness theorem, we must have $\Gamma \vdash^{c f} P$.

\section{Adding rewrite rules}

In this section, we show briefly how the result extends to deduction modulo in a straightforward way, provided the rewrite system verifies some conditions. We recall briefly the context of Deduction Modulo, but we suppose that the reader of this section is familiar with it, or at least with rewrite rules. For further informations, see for example $[5,4]$.

Definition 10. A term rewrite rule is a pair of terms $l \rightarrow r$ such that all the variables of $r$ appears in $l$.

A propositional rewrite rule is a pair of propositions $l \rightarrow r$ such that $l$ is atomic and all free variables of $r$ appears in $l$.

An example of a term rewrite rule is:

$$
x \times 0 \rightarrow 0
$$

An example of a propositional rewrite rule is:

$$
x \times y=0 \rightarrow(x=0) \vee(y=0)
$$

In this case, we notice that an atomic proposition can rewrite on a non-atomic proposition.

A rewrite system $\mathcal{R}$ is a set of propositional and term rewrite rules.

The deduction system is transformed in such a way that active propositions should be equal modulo the rewrite system considered. For example the new axiom rule will be:

$$
\overline{\Gamma, A \vdash_{\mathcal{R}} B} \text { axiom, with } A \equiv_{\mathcal{R}} B
$$

All definitions are transformed in a straightforward way, using cut-free provability modulo the rewrite rules $\vdash_{\mathcal{R}}^{c f}$ instead of cut-free provability $\vdash^{c f}$.

We introduce the notion of the validity of a rewrite system in a Kripke Structure. 
Definition 11. A rewrite system $\mathcal{R}$ is valid in a Kripke Structure iff the following property for any world $\alpha$ and propositions $A, B$ holds:

$$
\text { if } A \equiv_{\mathcal{R}} B \text { then } \alpha \Vdash A \Leftrightarrow \alpha \Vdash B
$$

When a Kripke Structure validates a rewrite system $\mathcal{R}$, we write the forcing relation $\Vdash_{\mathcal{R}}$.

We check that, given a confluent rewrite system, the proof of soundness theorem (w.r.t. Kripke Structure in which the rewrite system is valid), the completion process of section 3.1, and the proposition 7 still hold.

The only stage that differs from the former proof of the cut-elimination theorem is the construction of the Kripke Structure for $A$-complete, $A$-consistent theories that admit $A$-Henkin witnesses. Indeed, since the expressiveness of deduction modulo goes beyond first-order, we must have a stage in which the logical complexity appears.

So for different kinds of rewrite systems, we will have different model constructions. In some cases, these constructions can directly be derived from that described in section 4 , as we will shall see now.

\subsection{An order condition}

We will prove the cut-elimination theorem for all the rewrite systems verifying the following order condition. We consider a confluent rewrite system and a well-founded order $\prec$ such that:

- if $P \rightarrow_{\mathcal{R}} Q$ then $Q \prec P$.

- if $A$ is a subformula of $B$ then $A \prec B$.

This order condition was first introduced by Stuber [3] for proving completeness of Resolution Modulo (ENAR) with respect to Classical Sequent Calculus Modulo. Since we have this order, we can show that the rewrite system is normalizing, in the sense that every term has a normal form.

The Kripke Structure considered is the same as that of the proof of theorem 8 , which worlds are $A$-complete, $A$-consistent theories that admit $A$-Henkin witnesses, ordered by inclusion. The only slight difference is in the definition of the forcing relation $\Vdash_{\mathcal{R}}$. We first define it on normal atoms:

$$
\Gamma \Vdash_{\mathcal{R}} B \text { iff } B \in \Gamma
$$

We extend $\Vdash_{\mathcal{R}}$ on propositions following clauses $2-7$ of the definition 4 , and on non-normal atoms by $\Gamma \Vdash_{\mathcal{R}} B$ if $\Gamma \Vdash_{\mathcal{R}} B \downarrow$. This has to be done simultaneously.

The definition is well founded, since the order is well-founded, and at every step, we decrease the order.

We yet have to check that we really defined a forcing relation. The only point to present a difficulty is the first axiom of a forcing relation. Indeed, if an atom $A$ 
is non-normal, this is not self-evident to prove that $\Gamma \Vdash_{\mathcal{R}} A$ implies $\Delta \Vdash_{\mathcal{R}} A$ for $\Delta \supseteq \Gamma$. As usual, we have to show a more general result, that for any proposition $P$, for any $\Delta \supseteq \Gamma$ :

$$
\Gamma \Vdash_{\mathcal{R}} P \text { implies } \Delta \Vdash_{\mathcal{R}} P
$$

This is done by a straightforward induction over the well-founded order, rewriting non-normal atoms into their normal form.

Once we have the fact that we really have constructed a Kripke Structure, we remark that this is a Kripke Structure for the rewrite system. This is true by construction on the atoms, and we extend it to any proposition by induction over the proposition structure.

The last point to prove is that $\Gamma \Vdash_{\mathcal{R}} \Gamma$ and $\Gamma \nVdash_{\mathcal{R}} P$ (when $\Gamma$ is $P$-consistent). This is done exactly in the same way as in section 4 .

So, by the very same arguments as in section 4 the cut-elimination theorem holds for confluent rewrite systems compatible with a well-founded order. As an example, the following rewrite system is compatible with such an order:

$$
\begin{aligned}
x * y=0 & \rightarrow_{\mathcal{R}}(x=0) \vee(y=0) \\
x * 0 & \rightarrow_{\mathcal{R}} 0 \\
x+0 & \rightarrow_{\mathcal{R}} x
\end{aligned}
$$

in a general way, all the confluent, terminating, quantifier-free rewrite systems described in [5] are compatible with such an order. Stuber in [3] gives a more detailed example.

\subsection{A non-normalizing theory}

In this section, we transform a result of Dowek and Werner, that found a confluent and terminating rewrite system that doesn't enjoys the cut-elimination property. Here, we exhibit a confluent terminating rewrite system that enjoys cut-elimination, but that doesn't have proof normalization.

In [5], a non-normalizing confluent terminating rewrite system is presented. It is defined by the following rule, with $y \simeq z$ standing for $\forall x(y \in x \Rightarrow z \in x)$ :

$$
R \in R \rightarrow_{\mathcal{R}} \forall y(y \simeq R \Rightarrow \neg y \in R)
$$

Modulo this rewrite rule, we can prove both sequents $R \in R \vdash_{\mathcal{R}}^{c f}$ and $\vdash_{\mathcal{R}}^{c f}$ $R \in R$, so we can prove, using the cut rule, the sequent $\vdash_{\mathcal{R}}$ (the rewrite system is then inconsistent).

The idea is to modify slightly this rule, to get a consistent rewrite system, that we call $\mathcal{R}$ :

$$
R \in R \rightarrow_{\mathcal{R}} \forall y(y \simeq R \Rightarrow(y \in R \Rightarrow C))
$$


The same derivations lead this time to proofs of $R \in R \vdash_{\mathcal{R}}^{c f} C$ and of $\vdash_{\mathcal{R}}^{c f}$ $R \in R$. Proof terms are the same as in [5]. These two proofs can be combined with a cut and we get a proof of the sequent $\vdash_{\mathcal{R}} C$. We cannot eliminate the cut by the normalization method, because applying one proof term to the other leads by reductions to the same proof term. And in fact, any reduction-based cut-elimination will fail, since $\vdash_{\mathcal{R}}^{c f} C$ is not provable (what kind of rule could be the first rule, if not the cut rule?).

So, the rewrite system $\mathcal{R}$ doesn't normalize.

Let's now replace in (1) $C$ by a well-known intuitionistic tautology: $A \Rightarrow A$. We get a new set of rewrite rules $\mathcal{R}^{*}$. Of course, we can prove $\vdash_{\mathcal{R}^{*}}^{c f} A \Rightarrow A$ without the cut rule. But the former analysis is still valid, any normalization process fails if we try to eliminate cut from the following proof:

$$
\frac{R \in R \vdash_{\mathcal{R}^{*}}^{c f} A \Rightarrow A \quad \vdash_{\mathcal{R}^{*}}^{c f} R \in R}{\vdash_{\mathcal{R}^{*}} A \Rightarrow A} \text { cut }
$$

In fact, a normalization procedure can't make the difference between the two rewrite rules (with $C$ and with $A \Rightarrow A$ ), since proof terms are exactly the same in the two cases.

So this rewrite system doesn't enjoy normalization, however, we here show that it has the cut-elimination property, using the completeness method:

Proposition 12. The sequent calculus modulo $\mathcal{R}^{*}$ admits cut.

Proof. The principle is the same as in previous sections: we first prove the completeness theorem. Given a $B$-complete, consistent theory $\Gamma$, we construct a Kripke Structure that validates $\mathcal{R}^{*}$, and a node forcing $\Gamma$ and not forcing $B$.

The Kripke structure is defined as usual: $K$ is the set of all $C$-complete, consistent theories admitting $C$-Henkin witnesses, for some $C$, both expressed in one of the languages $\mathcal{L}_{i} . K$ is ordered by inclusion, and the domain $D(\Gamma)$ is the closed terms of $\mathcal{L}_{i}$.

The forcing relation is defined on atoms, no matter whether $\Delta, D \vdash_{\mathcal{R}^{*}}^{c f}$ or not:

$$
\Delta \Vdash D \text { iff } \Delta \vdash_{\mathcal{R}^{*}}^{c f} D
$$

This is extended over all the propositions. With this method, we are sure that we define a Kripke Structure. We check, as in section 4, that $\Gamma \Vdash \Gamma$ and that $\Gamma \nVdash B$ (when $\Gamma$ is $B$-consistent).

It remains yet to prove that we have defined a Kripke Structure for the rewrite rule. All we have to check is that the interpretation of $R \in R$ and of $\forall y(y \simeq R \Rightarrow(y \in R \Rightarrow(A \Rightarrow A)))$ is the same for any world $\Delta$.

Since $\vdash_{\mathcal{R}^{*}}^{c f} R \in R$, we have for any world $\Delta \Vdash R \in R$ (this is an atomic proposition). It remains to prove that $\Delta \Vdash \forall y(y \simeq R \Rightarrow(y \in R \Rightarrow(A \Rightarrow A)))$. 
Let $\Delta^{\prime} \supseteq \Delta$, and $t \in D\left(\Delta^{\prime}\right)$. Moreover, suppose $\Delta^{\prime} \Vdash t \simeq R$. We now have to prove $\Delta^{\prime} \Vdash t \in R \Rightarrow(A \Rightarrow A)$. This is trivial since $\Gamma^{\prime} \Vdash A \Rightarrow A$ for any $\Gamma^{\prime}$.

So the Kripke Structure constructed is a Kripke Structure for $\mathcal{R}^{*}$, the completeness theorem is proved and the announced result holds: this rewrite system enjoys cut-elimination.

The key to understand this result is that while proving the cut-elimination theorem we strongly need a semantic information, namely: $A \Rightarrow A$ is an intuitionistic tautology. This information is of course not available when defining a proof reduction process, and when trying to prove the termination of it. Another point that should be stressed is that the cut-free proof has nothing to do with the original proof.

\section{Conclusion and further work}

We have shown how to get the cut-elimination theorem by semantic methods, proving completeness of the cut-free intuitionistic calculus modulo with respect to Kripke Structures. Then we showed how this result extends to Deduction Modulo for an order condition on the rewrite system.

In our study of the links between proof normalization and cut admissibility,we have found a counterexample to the fact that proof normalization is equivalent to the redundancy of the cut rule. In [11], Okada gives a hint about a correspondence between his method (for Higher-Order Logic) and Girard's Reducibility Candidates, but doesn't gives any further information. We think that thanks to our negative result, there is no way to give such a correspondence without giving more information that we get with our model construction.

We should extend the semantic cut-elimination result to other theories modulo, such as the positive theories of [5], or to the formulation of Higher-Order Intuitionistic Sequent Calculus in Deduction Modulo. Then, it seems that one could add positive rules to the order condition, preserving the cut-elimination theorem. Finally, we can try to bridge the gap between semantic and syntactic proofs.

\section{References}

1. Gentzen, G.: Untersuchungen über das logische Schliessen. Mathematische Zeitschrift 39 (1934) 176-210, 405-431

2. Bachmair, L., Ganzinger, H.: 11. In: Associative-commutative superposition. Kluwer (1998) 353-397

3. Stuber, J.: A model-based completeness proof of extended narrowing and resolution. In: First International Joint Conference on Automated Reasoning (IJCAR2001). Volume 2083 of LNCS., Springer (2001) 195-210

4. Dowek, G., Hardin, T., Kirchner, C.: Theorem proving modulo. Journal of Automated Reasoning 31 (2003) 33-72 
5. Dowek, G., Werner, B.: Proof normalization modulo. The Journal of Symbolic Logic 68 (2003) 1289-1316

6. Prawitz, D.: Hauptsatz for higher order logic. The Journal of Symbolic Logic 33 (1968) 452-457

7. Takahashi, M.o.: A proof of cut-elimination theorem in simple type-theory. Journal of the Mathematical Society of Japan 19 (1967) 399-410

8. Andrews, P.B.: Resolution in type theory. The Journal of Symbolic Logic $\mathbf{3 6}$ (1971) 414-432

9. Troelstra, A.S., Schwichtenberg, H.: Basic Proof Theory. Cambridge University Press (1996)

10. De Marco, M., Lipton, J.: Cut elimination and completeness in church's intuitionistic theory of types. To appear (2003)

11. Okada, M.: A uniform semantic proof for cut-elimination and completeness of various first and higher order logics. Theoretical Computer Science 281 (2002) 471-498

12. Troelstra, A.S.: Metamathematical Investigation of Intuitionistic Arithmetic and Analysis. Springer-Verlag (1973)

13. Rasiowa, H., Sikorski, R.: The mathematics of metamathematics. PWN, Polish Scientific Publishers, Warsaw (1963)

14. Hermant, O.: A model-based cut elimination proof. 2nd St-Petersburg Days of Logic and Computability (2003)

15. Kripke, S.: Semantical analysis of intuitionistic logic. In Crossley, J.N., Dummett, M.A.E., eds.: Formal systems and recursive function. North-Holland (1965) 92-130

16. Troelstra, A.S., van Dalen, D.: Constructivism in Mathematics, An Introduction. North-Holland (1988)

17. Szabo, M.E., ed.: Collected Papers of Gerhard Gentzen. Studies in Logic and the Foundation of Mathematics. North Holland (1969) 